\title{
Gestão e Humanização do Serviço de Odontologia na Unidade de Saúde
}

Diego Fernando de Carvalho Alves ${ }^{I}$; Susanne Pinheiro Costa e Silva ${ }^{2}$

\begin{abstract}
Resumo: O presente estudo visa desvelar as publicações científicas que tratem sobre humanização e gestão de serviços odontológicos. Trata-se de uma revisão sistemática de literatura, na qual foram analisadas publicações disponíveis nas bases de dados LILACS, SciELO e Biblioteca Virtual da Saúde. Os termos utilizados para busca foram os descritores "Gestão", "Humanização da assistência" e "Saúde Bucal". Foram selecionadas 10 publicações, resultando em dois eixos de discussão: "Práticas ideais de humanização e gestão" e "Possibilidades para implementação". As publicações emergem para dificuldades da rede pública, principalmente as estruturais e de recursos humanos. A gestão deve ter o compromisso de qualificar permanentemente os profissionais da rede, evitando que o atendimento em odontologia não seja humanizado e incentivando a promoção à saúde e prevenção de doenças relacionadas. Faz-se necessário, ainda, o fortalecimento das redes de média e alta complexidade, visando o suporte adequado às demandas identificadas na atenção básica que necessitam de referência
\end{abstract}

Descriptores: Gestão em saúde; Humanização da assistência; Saúde Bucal.

\section{Humanization and Management of Dental Services at the Health Unit}

\begin{abstract}
The present study aims to unveil scientific publications dealing with humanization and management of dental services. It is a systematic review of the literature, in which publications available in the LILACS, SciELO and Virtual Health Library databases were analyzed. The terms used to search were the descriptors "Management", "Humanization of care" and "Oral Health ". Ten publications were selected, resulting in two discussion axes: "Ideal practices of humanization and management" and "Implementation Possibilities". The publications emerge for public network difficulties, mainly structural and human resources. The management must be committed to permanently qualify network professionals, avoiding that dental care is not humanized and encouraging health promotion and prevention of related diseases. It is also necessary to strengthen the networks of medium and high complexity, aiming at the adequate support to the demands identified in the basic attention that need reference.
\end{abstract}

Keywords: Health management; Humanization of care; Oral Health.

\section{Introdução}

No campo da saúde pública no Brasil, encontra-se atualmente um cenário repleto de discussões voltadas aos mais variados temas, dentre eles o acolhimento e a humanização do atendimento em saúde pública que, embora já tenha suscitado muitas discussões, ainda existe muito a ser estudado (CARVALHO, 2013).

\footnotetext{
${ }^{1}$ Graduação em andamento em Odontologia. Associação Caruaruense de Ensino Superior, ASCES, Brasil. Contato: drdiegocarvalho@gmail.com

${ }^{2}$ Doutora pelo Programa de Pós Graduação em Psicologia da Universidade Federal do Espírito Santo - UFES. Possui Mestrado em Enfermagem - Atenção à Saúde pela Universidade Federal do Rio Grande do Norte (UFRN) e graduação em Enfermagem pela Universidade Estadual da Paraíba (UEPB). Docente efetiva do Mestrado em Psicologia UNIVASF - disciplina Metodologia da Pesquisa em Psicologia. Vicecoordenadora do Colegiado de Enfermagem. Docente da Especialização Gestão em Saúde UNIVASF. Contato: susanne.pc@gmail.com
} 
Neste trabalho, procuramos enveredar pelas questões abordadas pela literatura disponível, voltadas ao atendimento humanizado nas unidades básicas de saúde, mais precisamente no que se refere à melhoria da qualidade do atendimento odontológico. Desde o início do programa Brasil Sorridente, em meados de 2004, há na gestão do Sistema Único de Saúde (SUS) uma preocupação com a melhoria do atendimento odontológico oferecido nas unidades básicas de saúde (UBS), no tocante ao acolhimento e manejo do usuário de forma mais humanizada.

Segundo Rios (2009), o atendimento humanizado é um termo que rememora movimentos de recuperação de valores sociais humanos que foram sendo esquecidos ao longo dos anos, principalmente em tempos aonde a ética vem cada vez mais sendo deixada de lado. O termo humanização é de complexa definição, uma vez que refere-se a uma mudança de conduta que vai além das práticas clínicas e técnicas específicas de cada profissional, requerendo além disso uma mudança comportamental frente às demandas do usuário.

Humanizar significa dar condição humana a alguma ação ou atitude, humanar. Também quer dizer ser benévolo, afável, tratável. É realizar qualquer ato considerando o ser humano como um ser único e complexo, estando inerentes o respeito e a compaixão para com o outro (FERREIRA, 2009). Logo, pode-se afirmar que humanizar é, prioritariamente, no que depende dos profissionais de saúde, amenizar ou tornar suportável a realidade do usuário, que muitas vezes não tem conforto nenhum em seus relacionamentos, estilo de vida, entre outros, sendo esta situação na qual está inserido, muitas vezes, a causa do agravo em saúde para o qual busca solução.

Dada esta realidade, é imprescindível a implementação de práticas que possam fazer com que o atendimento às angustias do usuário do SUS sejam feitas de forma mais eficiente e eficaz, com o mínimo de morosidade, buscando uma maior resolutividade a cada problema encontrado (MOTA; SANTOS; MAGALHÃES, 2012).

É necessário também adequar o atendimento às linhas de cuidado existentes (da criança, do adolescente, do adulto, da mulher, da gestante, do idoso, do homem), dando vazão ao fluxo de usuários que procuram o serviço de saúde, atendendo com qualidade à demanda existente (COSTA et al., 2004). 
De acordo com Guerra et al. (2014), a equipe de odontologia, formada por pessoas servindo outra pessoa em situação de vulnerabilidade e fragilidade, deve entender a importância da humanização do atendimento ao paciente. Desse modo, em situações de atendimento público como aqueles realizados em unidades básicas de saúde, os gestores devem atuar de forma que os recursos disponíveis, sejam eles humanos ou de infraestrutura, contribuam para tal. A adequação do uso destes recursos dar-se-á mediante análise eficiente e correta aplicação dos conhecimentos existentes, bem como a proposição de novas estratégias pela e de gestão.

Partindo deste pressuposto, o presente estudo visa desvelar as publicações científicas que tratem sobre humanização e gestão de serviços odontológicos. Espera-se que desperte o interesse de profissionais e gestores, norteando as suas práticas e auxiliando na adoção de medidas que propiciem uma funcionamento onde o acolhimento ao usuário e humanização da assistência em saúde sejam rotina.

\section{Método}

O presente estudo teve como proposta executar uma revisão da literatura sistemática através do levantamento e análise de textos, documentos oficiais e trabalhos acadêmicos (teses, dissertações, monografias e publicações em periódicos) publicados nas bases de dados LILACS, SciELO e ainda, Biblioteca Virtual da Saúde (BVS). Os termos utilizados para busca foram os descritores "Gestão", "Humanização da assistência" e "Saúde Bucal”. Os artigos, textos e documentos oficiais foram selecionados utilizando os seguintes critérios: Artigos e textos completos; publicações nos últimos 5 anos; escritos em língua portuguesa e inglesa.

$\mathrm{Na}$ base de dados Scientific Eletronic Library Online (SciElo), ao utilizarmos os filtros dos descritores escolhidos foram encontradas 11 referências, das quais foram selecionadas 4 por terem correlação direta com o tema proposto. Na BVS, encontramos 6 publicações, sendo 4 selecionadas. Na base de dados LILACS foram encontradas 4 publicações, sendo selecionadas 2.

Foram pré-selecionados 5 documentos oficiais dos quais apenas 1 foram utilizados em nosso trabalho, por fazer referência em seu conteúdo ao assunto abordado neste trabalho. 
Analisou-se os títulos das publicações, excluindo-se aquelas que não se adequaram à proposta. Posteriormente, os resumos foram lidos, excluindo ainda os artigos que diferiam do tema proposto. Finalmente, foram lidas na íntegra e consideradas para fins desta revisão todas as publicações que se adequaram aos critérios de inclusão propostos (Tabela 1).

Tabela 1: Distribuição das publicações encontradas, de acordo com suas bases de dados de origem.

\begin{tabular}{c|c|c|c|c}
\cline { 2 - 5 } & SciELO & BVS & LILACS & $\begin{array}{c}\text { Documentos } \\
\text { Oficiais }\end{array}$ \\
\hline Encontrados & 11 & 06 & 04 & 05 \\
\hline Utilizados & 04 & 04 & 01 & 01 \\
\hline
\end{tabular}

Fonte: autores

Incluiu-se entre os trabalhos escolhidos para análise, ainda, o HumanizaSUS - Política Nacional de Humanização (2003), que apesar de fugir de critérios como "ano limite de publicação", é parte fundamental da estrutura da proposta do trabalho.

\section{Resultados e Discussão}

A partir dos estudos encontrados, estabeleceu-se o diálogo inicial do tema gestão, humanização e serviço público de odontologia. Todos aqueles selecionados e analisados para a construção desta revisão estão apresendados na Tabela 2.

Tabela 02: Quadro descritivo dos artigos selecionados

\begin{tabular}{|c|c|c|c|c|}
\hline $\begin{array}{l}\text { Proce- } \\
\text { dência }\end{array}$ & Título da publicação & Autores & Fonte & Considerações/ Temática \\
\hline SciELO & $\begin{array}{l}\text { A integralidade no } \\
\text { cotidiano da atenção à } \\
\text { saúde bucal: Revisão } \\
\text { de literatura. }\end{array}$ & $\begin{array}{l}\text { SANCHEZ, H. } \\
\text { P.; WERNECK, } \\
\text { M. A. F.; } \\
\text { AMARAL, J. H. } \\
\text { L.; FERREIRA, } \\
\text { E. F. A }\end{array}$ & $\begin{array}{l}\text { Trab. Educ. } \\
\text { Saúde, Rio de } \\
\text { Janeiro, v. } 13 \\
\text { n. 1, p. } 201- \\
\text { 214, jan./abr. } \\
2015 .\end{array}$ & $\begin{array}{l}\text { Considera que para a } \\
\text { possibilidade de uma gestão } \\
\text { humanizada, existe a } \\
\text { necessidade de que os } \\
\text { profissionais tenham uma visão } \\
\text { ampliada da saúde, percebendo } \\
\text { os usuários a partir de sua } \\
\text { totalidade biopsicossocial. }\end{array}$ \\
\hline
\end{tabular}




\begin{tabular}{|c|c|c|c|c|}
\hline LILACS & $\begin{array}{l}\text { A visão do usuário } \\
\text { sobre o serviço público } \\
\text { odontológico e a } \\
\text { autopercepção em } \\
\text { saúde. }\end{array}$ & AYACH, C. & $\begin{array}{c}\text { Tese } \\
\text { (Doutorado } \\
\text { em } \\
\text { Odontologia } \\
\text { Preventiva) } \\
\text { Universidade } \\
\text { Estadual } \\
\text { Paulista - } \\
\text { UNESP. } \\
\text { 2015, } \\
\text { Araçatuba } \\
\end{array}$ & $\begin{array}{l}\text { Destaca a importância da } \\
\text { melhoria do acesso ao serviço de } \\
\text { saúde, para uma perspectiva de } \\
\text { experiência mais satisfatória } \\
\text { pelo usuário. }\end{array}$ \\
\hline SciELO & $\begin{array}{l}\text { Características ideais } \\
\text { do cirurgião-dentista na } \\
\text { estratégia saúde da } \\
\text { família. }\end{array}$ & $\begin{array}{l}\text { SOARES, E. F.; } \\
\text { REIS, } \\
\text { S. C. G. B.; } \\
\text { FREIRE, M. C. } \\
\text { M. }\end{array}$ & $\begin{array}{c}\text { Trabalho } \\
\text { Educação e } \\
\text { Saúde. Trab. } \\
\text { Educ. Saúde, } \\
\text { Rio de } \\
\text { Janeiro, v. } 12 \\
\text { n. 2, p. 327- } \\
\text { 341,maio/ago. } \\
\text { 2014. }\end{array}$ & $\begin{array}{l}\text { Ressalta o anseio dos usuários } \\
\text { pela presensa de profissionais de } \\
\text { fácil socialização, buscando } \\
\text { termos como: humildade, } \\
\text { companheirismo e receptividade } \\
\text { para definir características } \\
\text { ideais. }\end{array}$ \\
\hline SciELO & $\begin{array}{c}\text { A saúde Pública no } \\
\text { Brasil. }\end{array}$ & $\begin{array}{l}\text { CARVALHO, } \\
\text { G. A. }\end{array}$ & $\begin{array}{c}\text { Estudos } \\
\text { Avançados. } \\
\text { Vol 27. São } \\
\text { Paulo. 2013. }\end{array}$ & $\begin{array}{l}\text { Caracteriza o acolhimento do } \\
\text { usuário como um dos principais } \\
\text { fatores para uma gestão } \\
\text { humanizada. }\end{array}$ \\
\hline BVS & $\begin{array}{c}\text { Humanização em saúde } \\
\text { e a odontologia. }\end{array}$ & $\begin{array}{l}\text { CARNEIRO } \\
\text { NETO, J. N. C.; } \\
\text { CORDEIRO, T. } \\
\text { M. S. C.; } \\
\text { FALCÃO, M. } \\
\text { M. L. }\end{array}$ & $\begin{array}{l}\text { Rev. Bras. } \\
\text { Pesq. Saúde, } \\
\text { Vitória, 16(2): } \\
\text { 130-138, abr- } \\
\text { jun, 2014. }\end{array}$ & $\begin{array}{l}\text { Defende que humanizar o } \\
\text { atendimento não se relaciona } \\
\text { apenas às questões do } \\
\text { atendimento clínico, perpassa } \\
\text { pela compreensão das angústias } \\
\text { e incertezas do indivíduo, } \\
\text { dando-lhe apoio e atenção } \\
\text { permanente na busca da solução } \\
\text { do problema. }\end{array}$ \\
\hline BVS & $\begin{array}{l}\text { Humanização no } \\
\text { atendimento } \\
\text { odontológico: } \\
\text { acolhimento da } \\
\text { subjetividade dos } \\
\text { pacientes atendidos por } \\
\text { alunos de graduação } \\
\text { nos campos de estágio. }\end{array}$ & $\begin{array}{l}\text { MOTA, L. Q.; } \\
\text { SANTOS, T. } \\
\text { A.; } \\
\text { MAGALHÃES, } \\
\text { D. B. L. }\end{array}$ & $\begin{array}{l}\text { Revista } \\
\text { Brasileira de } \\
\text { Ciências da } \\
\text { Saúde. João } \\
\text { Pessoa, v. } 16 \\
\text { n. 4, p } 537- \\
\text { 544. } 2012 \text {. }\end{array}$ & $\begin{array}{l}\text { A satisfação do usuário é um dos } \\
\text { fatores que determina a } \\
\text { qualidade do atendimento nos } \\
\text { serviços de saúde, e por meio } \\
\text { dela se obtêm dados sobre } \\
\text { expectativas e se buscam } \\
\text { soluções para a melhoria do } \\
\text { serviço prestado. }\end{array}$ \\
\hline BVS & $\begin{array}{l}\text { Gestão em redes e } \\
\text { governança local. }\end{array}$ & $\begin{array}{l}\text { GRANJA, G. } \\
\text { F.; ZOBOLI, E. } \\
\text { L. C. P. }\end{array}$ & $\begin{array}{l}\text { O Mundo da } \\
\text { Saúde, São } \\
\text { Paulo, v.36 } \\
\text { n.3 p. 494- } \\
501,2012 \text {. }\end{array}$ & $\begin{array}{l}\text { Considera fundamental o } \\
\text { estabelecimento de uma } \\
\text { governança compartilhada entre } \\
\text { os pontos da rede (atenção } \\
\text { primária e demais níveis) para a } \\
\text { obtenção de resultados } \\
\text { satisfatórios às necessidades de } \\
\text { saúde, em um cuidado } \\
\text { humanizado. }\end{array}$ \\
\hline $\begin{array}{l}\text { Ministério } \\
\text { da Saúde }\end{array}$ & $\begin{array}{c}\text { HumanizaSUS Política } \\
\text { Nacional de } \\
\text { Humanização } \\
\end{array}$ & BRASIL & $\begin{array}{l}\text { Ministério da } \\
\text { Saúde }\end{array}$ & $\begin{array}{l}\text { Enumera e discute os princípios } \\
\text { norteadores da política nacional } \\
\text { humanização (PNH). }\end{array}$ \\
\hline
\end{tabular}




\begin{tabular}{|c|c|c|c|c|}
\hline SciELO & $\begin{array}{c}\text { Percepções de usuários } \\
\text { e trabalhadores de } \\
\text { saúde sobre a } \\
\text { implantação do } \\
\text { acolhimento em uma } \\
\text { unidade de saúde em } \\
\text { Porto Alegre-RS, } \\
\text { Brasil }\end{array}$ & $\begin{array}{l}\text { NEVES, M.; } \\
\text { PRETTO, S. L.; } \\
\text { ELY, H. C. }\end{array}$ & $\begin{array}{c}\text { Revista de } \\
\text { Odontologia } \\
\text { da UNESP. } \\
\text { 42(5): } 364- \\
\text { 371, Sept-Oct; } \\
\text { 2013. }\end{array}$ & $\begin{array}{l}\text { Conceitua a humanização como } \\
\text { termo que vai além dos cuidados } \\
\text { para com o outro no } \\
\text { atendimento, a partir do resgate } \\
\text { do sujeito e da satisfação de suas } \\
\text { necessidades mais essenciais, } \\
\text { que o mantém como um ser que } \\
\text { trabalha, vive e se faz } \\
\text { socialmente, com direitos e } \\
\text { deveres, com consciência e } \\
\text { liberdade de escolha sobre sua } \\
\text { própria vida. }\end{array}$ \\
\hline $\begin{array}{l}\text { Archives } \\
\text { of health } \\
\text { investigati } \\
\text { on }\end{array}$ & $\begin{array}{l}\text { Reflexões sobre o } \\
\text { conceito de } \\
\text { atendimento } \\
\text { humanizado em } \\
\text { Odontologia }\end{array}$ & $\begin{array}{l}\text { GUERRA, C. } \\
\text { T.; BERTOZ, } \\
\text { A. P. M.; } \\
\text { FAJARDO, R. } \\
\text { S.; REZENDE, } \\
\text { M. C. R. A. }\end{array}$ & $\begin{array}{l}\text { Archives of } \\
\text { Health } \\
\text { Investgation } \\
(2014) 3(6) \text { : } \\
31-36 ; 2014 .\end{array}$ & $\begin{array}{l}\text { Para que haja a humanização do } \\
\text { atendimento ao paciente, é } \\
\text { necessário um relacionamento } \\
\text { interno de confiança entre a } \\
\text { equipe, público e gestores. O } \\
\text { cotidiano odontológico deve } \\
\text { considerar aspectos } \\
\text { afetivos, cognitivos e } \\
\text { psicomotores de cada paciente. }\end{array}$ \\
\hline
\end{tabular}

Fonte: autores

Para facilitar a análise das publicações selecionadas em nosso trabalho, dividimos basicamente em dois eixos. O primeiro, composto por aquelas onde há apenas uma caracterização das práticas ideais de humanização e gestão; e o segundo, que basicamente refere-se às características de possibilidade para a sua implementação, tratando-as como ponto crucial e como um desafio para a obtenção de uma gestão humanizada.

\section{Práticas Ideais de Humanização e Gestão}

O HumanizaSUS (BRASIL, 2003) considera que a aplicação da Política Nacional de Humanização (PNH) pressupõe uma atuação em eixos diversificados, sendo eles: Eixo das Instituições; Educação permanente; Informação/comunicação; Atenção à saúde; Financiamento; Gestão da política. Estes eixos responsabilizam desde os gestores, equipes de saúde e até mesmo o usuário, no que compete ao feedback, seja ele positivo ou não, da sua experiência enquanto paciente de uma unidade de saúde.

Para a melhoria da atenção básica, o PNH propõe a individualização de projetos terapêuticos, inclusão da clientela (minimizando filas e hierarquizando os riscos), terapias que 
tragam a resolução dos agravos ao mesmo tempo que evitem desperdício dos insumos, estabelecimentos abertos à escuta da população para promoção de uma gestão participativa e, finalmente, requisita ambientes acolhedores e confortáveis, mas que mantenham a privacidade de cada usuário.

Não é raro observar que mesmo diante de recomendações já não tão recentes quanto à humanização do atendimento em odontologia, ainda é comum que o usuário procure o serviço odontológico por conta própria. Apenas uma minoria chega à unidade encaminhada por agente comunitário de saúde e, muito menos, pelo cirurgião-dentista, evidenciando que não há uma ação efetiva deste no planejamento de suas ações, especialmente no que se refere à organização das demandas nas unidades de saúde (AYACH, 2015).

Carvalho (2013) discorre sobre pontos necessários para a melhoria da saúde pública no Brasil, tratando a humanização como uma necessidade imediata para a reconstrução do modelo de assistência atual, que infelizmente ainda é baseado em práticas intervencionistas que generalizam e padronizam em excesso o atendimento ao usuário. Nessa realidade, muitas vezes, podemos encontrar indivíduos insatisfeitos com a assistência a qual têm acesso.

De acordo com os estudos de Sanchez et al. (2015), as práticas ideias para a efetivação da humanização no SUS pelo serviço de odontologia, são apontadas como o trabalho em equipe/atuação multiprofissional, atuação interdisciplinar e outras transversalidades. Deve-se considerar que a integralidade na prática é sempre uma construção local, com limites e possibilidades de cada equipe, recursos disponíveis, atuação dos gestores e/ou das cobranças dos usuários.

Somando-se a isso, o cirurgião-dentista, ao atuar em equipes das unidades básicas, deve saber dialogar e aceitar a comunidade como ela é. Pode haver lacunas na formação destes profissionais para atuar em comunidades mais pobres, sendo a capacidade algo a ser melhorado, já que com frequência há dificuldade deles em trabalhar com pessoas de condição socioeconômica mais baixa. Tanto o profissional de saúde bucal quanto os demais devem apresentar características condizentes com um modelo de cuidado em saúde integral e humanizado, que requer tanto competências técnicas quanto interpessoais (SOARES; REIS; FREIRE, 2014).

A correta percepção dessa gama de fatores subjetivos que influenciam nos agravos vivenciados por cada usuário, demanda a sensibilidade do profissional, mostrando que na 
unidade básica de saúde, além de competência técnica, necessita-se de socialização, comunicação e de ampla visão, enxergando o usuário como um ser biopsicossocial, inserido em uma realidade complexa, composta de muitas variáveis (SOARES; REIS; FREIRE, 2014).

Em contrapartida, a compreensão da realidade de cada indivíduo enquanto usuário do serviço também pode ser considerada como parte fundamental de uma assistência humanizada, uma vez que o tratamento tem início em questões que excedem em muito a clínica propriamente dita (CARNEIRO NETO; CORDEIRO; FALCÃO, 2014).

Ainda, considerando os resultados encontrados, pode-se sugerir que através do nível de satisfação do usuário, mensura-se a qualidade do serviço prestado, bem como a eficiência do sistema de referência e contra referência da rede (MOTA; SANTOS; MAGALHÃES, 2012).

É necessário avaliar periodicamente as ações oferecidas aos usuários do serviço de saúde, auxiliando na melhoria da atenção e das estratégias em andamento, o que permite avançar na prática de avaliação no cotidiano da gestão em saúde, instituindo mudanças necessárias e tomando o usuário o objeto central do processo de trabalho (AYACH, 2015).

\section{Possibilidades para Implementação}

A satisfação é uma conquista que precisa ainda ser trazida à realidade do cotidiano da gestão, como também da equipe de saúde bucal. Para tanto, informações trazidas nas publicações analisadas demonstram-se bastante pertinentes.

Tomando por base o sentido do termo humanização abordado no presente trabalho, no qual ambiente humanizado pressupõe unidade ou serviço de saúde organizado de forma a ter o usuário como centro do seu funcionamento (NEVES; PRETTO; ELY, 2013), pôde-se observar uma correlação direta entre a qualidade do acolhimento ao usuário no serviço e o relato de satisfação do mesmo.

Considerando que, segundo Ayach (2015), mais de um quarto dos usuários procuram o serviço devido a quadros de sintomatologia dolorosa aguda, é necessário compreender que o tempo de espera entre a procura e o atendimento torna-se um fator importante a ser trabalhado, além de que são, em sua grande maioria, mães de família, donas de casa e adultos jovens em plena atividade laboral. 
Diante do exposto, é perceptível um paradoxo entre a necessidade de resolutividade e fluidez no atendimento, causando a mecanização do mesmo e afastamento entre equipe e usuário devido à falta de entendimento profundo da realidade deste, e a necessidade de atendimento individualizado e acolhedor, que apesar dos bons resultados, gera uma certa morosidade no serviço, visto que a individualização da terapêutica requer conhecimento do quadro como um todo, que por sua vez leva tempo. Neves, Pretto e Ely (2013) referem que a resolutividade do serviço após a implantação do acolhimento no atendimento em odontologia ainda é um desafio a ser ultrapassado.

Faz-se necessária uma boa gestão, com governança compartilhada e melhor articulação entre os vários pontos da rede pública de assistência para que possa ser extraído o melhor de dois dos aspectos cruciais para o bom atendimento: assistência humanizada e individualizada e agilidade e fluidez mínimas para a resolução das angústias dos usuários em tempo hábil, diminuindo assim o seu sofrimento (GRANJA; ZOBOLI, 2012).

A construção de uma postura acolhedora e humanizada requer também formação e educação permanente dos profissionais, no intuito de sensibilizar a classe odontológica para a importância do cuidado em saúde preconizado pela $\mathrm{PNH}$, subsidiando novas práticas com enfoque nas tecnologias leves, focado na produção e promoção da saúde bucal, perpassando pela prevenção e culminando na reabilitação, quando necessária (NEVES; PRETTO; ELY, 2013; CARNEIRO NETO; CORDEIRO; FALCÃO, 2014).

Mesmo após muitos avanços, como a implantação da Saúde da Família, que preconiza o vínculo da assistência, ainda persistem práticas nas quais os usuários ocupam posição passiva nos serviços. É importante lembrar que estes devem exprimir as necessidades de saúde da comunidade, fortalecendo os processos educativos e a cidadania (GRANJA; ZOBOLI, 2012).

Dessa forma, urge a criação e efetivação de mecanismos democráticos, a partir de planejamento participativo, com transparência na gestão e organização das ações com base nas necessidades de saúde, e não apenas em interesses particulares. Esse tipo de gestão só é possível quando há uma relação de confiança e respeito mútuos, entre gestão, equipe e usuários. A gestão deve buscar entender as necessidades da equipe e sua clientela; a equipe deve ter conhecimento técnico e infraestrutura para realizar atendimento de qualidade; informações pertinentes para atualização dos planos de ação dos gestores; e, finalmente, o usuário com acesso a serviço individualizado e de qualidade, em tempo minimamente satisfatório, além de voz ativa para 
falar sobre seus anseios, informar sobre suas queixas e dar suas sugestões para a melhoria do atendimento (GRANJA; ZOBOLI, 2012; GUERRA et al., 2014).

\section{Considerações Finais}

É possível verificar que a literatura ainda apresenta pequeno número de publicações que tratam da gestão e humanização do serviço de saúde de odontologia na Unidade Básica de Saúde, e aquelas encontradas tratam, em sua maioria, de forma indireta sobre o tema.

As publicações emergem para dificuldades basicamente de infraestrutura da rede pública ou ainda comportamental por parte dos recursos humanos, já que uma das dificuldades mais relatadas referiu-se a relação interpessoal entre equipe de saúde bucal e usuários. É necessário entender que esta é parte da equipe de Saúde da Família, que deve priorizar o acolhimento e o atendimento humanizado.

Contudo, a presença de uma equipe capacitada tecnicamente, que participe de atividades de educação permanente em busca de melhorias para a sua atuação é algo que deve ser incentivado pela gestão, na tentativa de dirimir possíveis falhas que estejam ocorrendo ou possam vir a acontecer.

A partir dos dados apresentados, a gestão deve ter o compromisso de qualificar permanentemente os profissionais que fazem parte da sua rede, evitando que o atendimento em odontologia não seja humanizado e incentivando também a promoção à saúde e prevenção de doenças relacionadas. Somando-se a isso, deve garantir a resolutividade e disponibilidade de material para um trabalho de qualidade.

Faz-se necessário ainda o fortalecimento das ações no âmbito das unidades de média e alta complexidade, para que haja suporte adequado às demandas identificadas na atenção básica e que não possam ser resolvidas nesse nível de cuidado, impulsionado pelo sistema de referência e contra referência entre estes serviços. 


\section{Referências}

AYACH, C. A visão do usuário sobre o serviço público odontológico e a autopercepção em saúde. Tese (Doutorado em Odontologia Preventiva) Universidade Estadual Paulista - UNESP. 2015, Araçatuba - SP.

BRASIL, Ministério da Saúde. HumanizaSUS Política Nacional de Humanização: Documento para discussão. Brasília, 2003.

CARNEIRO NETO, J. N. C.; CORDEIRO, T. M. S. C.; FALCÃO, M. M. L. Humanização em saúde e a odontologia. Revista Brasileira Pesquisa em Saúde, Vitória, Vol. 16 n.2 p. 130$138,2014$.

CARVAlHO, G. A saúde Pública no Brasil. Estudos Avançados. Vol 27. São Paulo. 2013. Disponível em <http://www.scielo.br/scielo.php?pid=S010340142013000200002\&script=sci_arttext $>$.

COSTA, H.; SOLlA J.; SUASSUNA, A. Diretrizes da Política Nacional de Saúde Bucal. Brasília, Ministério da Saúde. 2004. Disponível em <http://bvsms.saude.gov.br/bvs/publicacoes/politica_nacional_brasil_sorridente.pdf >.

FERREIRA, A. B. de H. Novo Dicionário Aurélio da língua portuguesa. Editora Positivo. Curitiba: 2009.

GRANJA, G. F.; ZOBOLI, E. L. C. P. Gestão em redes e governança local. O Mundo da Saúde, São Paulo, v.36 n.3 p. 494-501, 2012.

GUERRA, C. T.; BERTOZ, A. P. M.; FAJARDO, R. S.; REZENDE, M. C. R. A. Reflexões sobre o conceito de atendimento humanizado em Odontologia. Archives of Health Investgation, Vol. 3 n.6 p. 31-36, 2014.

MOTA, L. Q.; SANTOS, T. A.; MAGALHÃES, D. B. L. Humanização no atendimento odontológico: acolhimento da subjetividade dos pacientes atendidos por alunos de graduação nos campos de estágio. Revista Brasileira de Ciências da Saúde. João Pessoa, v. 16 n. 4, p $537-544.2012$.

NEVES, M.; PRETTO, S. L.; ELY, H. C. Percepções de usuários e trabalhadores de saúde sobre a implantação do acolhimento em uma unidade de saúde em Porto Alegre-RS, Brasil. Revista de Odontologia da UNESP.Vol. 42 n.5 p. 364-371, 2013.

RIOS. I. C. Caminhos da Humanização na Saúde: Prática e Reflexão. São Paulo - SP. Áurea, 2009. 
SANCHEZ, H. P.; WERNECK, M. A. F.; AMARAL, J. H. L.; FERREIRA, E. F. A integralidade no cotidiano da atenção à saúde bucal: Revisão de literatura. Trab. Educ. Saúde, Rio de Janeiro, v. 13 n. 1, p. 201-214, jan./abr. 2015.

SOARES, E. F.; REIS, S. C. G. B.; FREIRE, M. C. M. Características ideais do cirurgiãodentista na estratégia saúde da família. Trabalho Educação e Saúde. Trab. Educ. Saúde, Rio de Janeiro, v. 12 n. 2, p. 327-341,maio/ago. 2014.

\section{Como citar este artigo (Formato ABNT):}

ALVES, Diego F.C.; COSTA e SILVA, Susanne P. Gestão e Humanização do Serviço de Odontologia na Unidade de Saúde. Id on Line Revista Multidisciplinar e de Psicologia, Maio de 2017, vol.11, n.36, p.1-12. ISSN: 19811179 .

Recebido: 04.06.2017

Aceito: 09.06. 2017 